\title{
Research of long distance temperature measuring instrument based on ADT70
}

\author{
Kang $\mathrm{Li}^{1,}$ a , Liang $\mathrm{Li}^{1}$, Shuren $\mathrm{Gao}^{2}$ and Tao Wang ${ }^{2}$ \\ ${ }^{1}$ Center For Hydrogeology And Environmental Geology Survey, CGS. Key Laboratory of Geological Environment \\ Monitoring Technology,MLR. Projects of Lanzhou and xining economic zone comprehensive geological \\ survey. BaoDing,071051, China \\ ${ }_{2}^{2}$ Petroleum Production Engineering Research Institute, Huabei Oilfield Company, CNPC, Renqiu, 062552, China
}

\begin{abstract}
This paper introduces a research methods of platinum thermal resistance signal conditioning module to long distance temperature measuring instrument. Mainly used ADT70 to turned the PT1000 platinum resistance temperature sensor resistance change when the temperature change into a voltage signal output.,And design the hardware circuit from several aspects,such as the lower cable interference and low power consumption, etc.This design can be used in underground water well water temperature measurement temperature measuring work and already be applied in 100 meters of temperature measurement range.
\end{abstract}

Keywords: PT1000; long distance temperature measurement; ADT70; single chip microcomputer; differential signal.

\section{Introduction}

In the field of geological survey and environmental protection, groundwater water temperature is a very important test parameters. At present, the well water temperature measuring instruments have many types, mainly uses the sensor of a p-n junction, platinum resistance, DS18B20, AD590, etc, they each have their own advantages and disadvantages, but In the aspect of remote temperature measurement, the performance of the platinum thermal resistance temperature measurement is the most stable[1].The temperature sensor precision of PT1000 higher than PT100,We use PT1000.

PT1000 is high precision temperature sensor, its value directly proportional with the change in temperature. When the temperature $0{ }^{\circ} \mathrm{C}, \mathrm{PT} 1000$ resistance is $1000 \mathrm{ohms}$, When the temperature is $100{ }^{\circ} \mathrm{C}$, the resistance of the PT1000 is about $1385.055 \mathrm{ohms}$, its value into uniform growth as temperatures rise. Its application scope is wide,can be measured - temperature of $200{ }^{\circ} \mathrm{C}$ to $650{ }^{\circ} \mathrm{C}[2]$.Its has many advantages,include high measurement accuracy, good stability, wide measuring range, etc.This research uses platinum thermal resistance signal conditioning module ADT70 to build temperature measuring circuit, and controlled by single chip microcomputer to realize the temperature measurement function.

${ }^{a}$ Corresponding author : 347266065@qq.com 


\section{Brief introduction of ADT70}

ADT70 is integrated platinum resistance signal conditioner, is produced by ADI company, the United States. Its internal includes symmetric current source, $2.5 \mathrm{~V}$ reference voltage source, instrumentation amplifier,Standby amplifier,Shut off the circuit etc.Using common diaphragm platinum resistance can measurement $-50{ }^{\circ} \mathrm{C} \sim+500{ }^{\circ} \mathrm{C}$ temperature, If use the high performance of platinum resistance, The upper limit of temperature measurement can reach $+1000{ }^{\circ} \mathrm{C}$, The measurement error is only $+/$ $1{ }^{\circ} \mathrm{C}$,ADT70 produced additional error can also be transferred to 0 after calibration.

ADT70 can use a single $+5 \mathrm{v}$ power supply, also can use double $+5 \mathrm{v}$ and $-5 \mathrm{v}$ power supply,inside it, there are two symmetrical current source, used to provide exciting current of $1 \mathrm{~mA}$ to standard resistance and platinum resistance. The internal instrumentation amplifier can enlarge the voltage difference on the standard resistance and platinum resistance into standard voltage signal, and the standard voltage signal is proportional to the degrees Celsius temperature[3].

ADT70 use the encapsulation of DIP-20 or SOIC20,Pin function is as follows:

$+\mathbf{U}_{\mathrm{S}}$ and $-\mathbf{U}_{\mathrm{S}}$ : Connect the positive and negative power supply respectively;

$\mathbf{U}_{\text {REFOUT: }}: 2.5 \mathrm{~V}$ reference voltage output;

SGND, AGND and DGND: sensor, analog and digital ground respectively;

BIAS: BIAS side, with the sub $U_{\text {REFOUT }}$ end, provide $2.5 \mathrm{~V}$ BIAS voltage for on chip current source, instrument amplifier, standby amplifier;

$\mathbf{I}_{\text {OUTA }}$ and $\mathbf{I}_{\text {OUTB }}$ : the 2 output ends of the current source;

NULLA and NULLB:the balance adjustment of a current source,adjust the two ends of the voltage, can guarantee the current source output two way equal to the size of the current,through on both ends of the $\mathrm{I}_{\text {OUTA }}$ and $\mathrm{I}_{\text {OUTB }}$ output.

RGA and RGB:the two connection section for instrumentation amplifier adjustable gain resistance;

SHUTDOWN: shut off the control side, when this end is low electricity at ordinary times, the chip is in standby mode;

$+\mathbf{I N}_{\mathbf{O A}},-\mathbf{I} \mathbf{N}_{\mathbf{O A}}$ :the inphase input and inverting input with standby amplifier;

OUT $_{\text {IA }}$ : the output of the instrumentation amplifier.

\section{The design of the temperature measuring system}

Temperature measurement system consists of three parts, respectively temperature measurement instrument, platinum thermalresistance sensor (including the signal conditioning circuit) and the transmission cables. The device mainly include PT1000 temperature sensor, ADT70, precision standard resistance,STC12C5410ADsingle chip microcomputerwith the AD conversion function,LCM045A LCD, Voltage chipNCP551 etc. The system structure diagram as shown in figure 1.

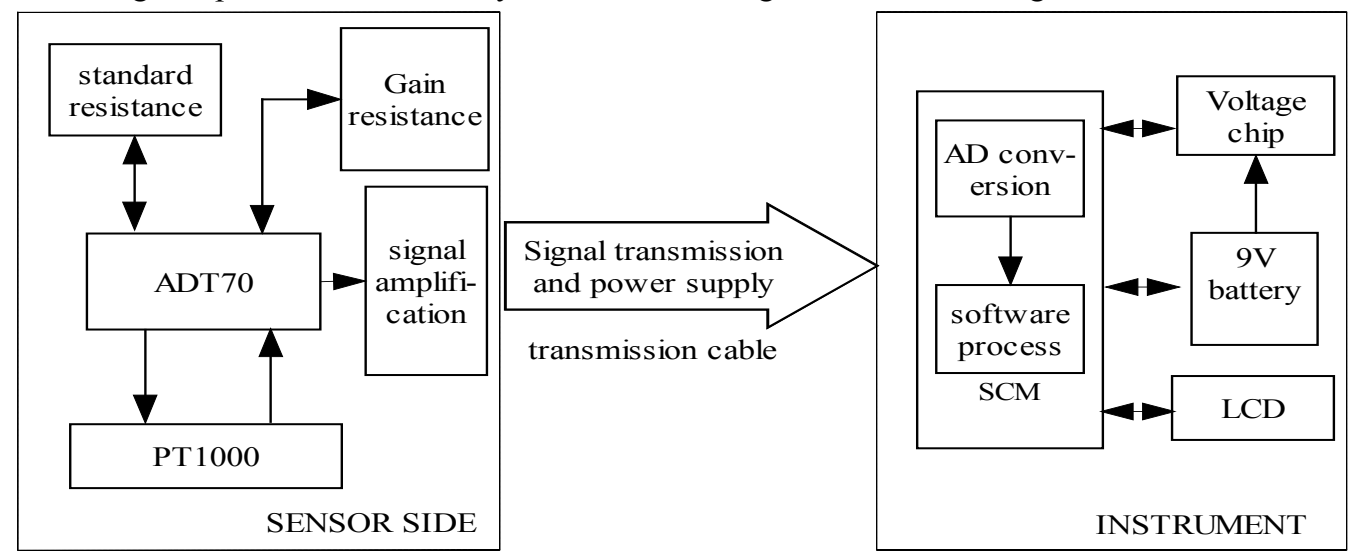

Figure 1.The system structure diagram 


\subsection{Design of sensors on the circuit}

In the process of measurement, due to often use a long cable, even can reach a few hundred meters, voltagecurrent losshave a significant impact to measure. This can not be ignored [3-4].

The sensor adopts two wire PT1000 platinum resistances, high precision resistor applies $1 \mathrm{k}$ resistor. ADT70 Internal current source provide two road equal size of current to Standard resistance and platinum thermal resistance, the voltage on the standard resistance and platinum thermal resistance Was sent to the instrumentation amplifier in the phase input and the inverting input. After the voltage amplifier, $\mathrm{OUT}_{\mathrm{IA}}$ side can acquirevoltage signal, and is proportional to the measured temperature.The instrumentation amplifier gain is decided by the resistance tolerance of RGA and RGB end connection [5].As shown in figure 2.

In figure $2, R_{1}=1 \mathrm{~K} \Omega, \mathrm{R}_{2}$ is $\mathrm{PT} 1000$, When the temperature of $0^{\circ} \mathrm{C}, \mathrm{R}_{2}=1 \mathrm{~K} \Omega$.When $\mathrm{R}_{3}=49.9 \mathrm{~K} \Omega$,Instrumentation amplifier gain is $\mathrm{K}_{\mathrm{V}}=1.3$. excitation current $\mathrm{I}_{\mathrm{o}}=1 \mathrm{~mA}$. When the temperature of $0^{\circ} \mathrm{C}, \mathrm{R}_{2}=\mathrm{R}_{1}=1 \mathrm{~K} \Omega$, When the temperature changes, $\mathrm{R}_{1}$ Resistance is constant, $\mathrm{R}_{1}=1 \mathrm{~K}$, but $\mathrm{R} 2$ change into $\left(R_{2}+\Delta R\right)$, at this time the input of the instrumentation amplifier is $\operatorname{Io}\left(R_{2}-R_{1}\right)$, the output voltage is $\mathrm{Uo}=\mathrm{K}_{\mathrm{v}} * \operatorname{Io}\left(\mathrm{R}_{2}-\mathrm{R}_{1}\right)$.

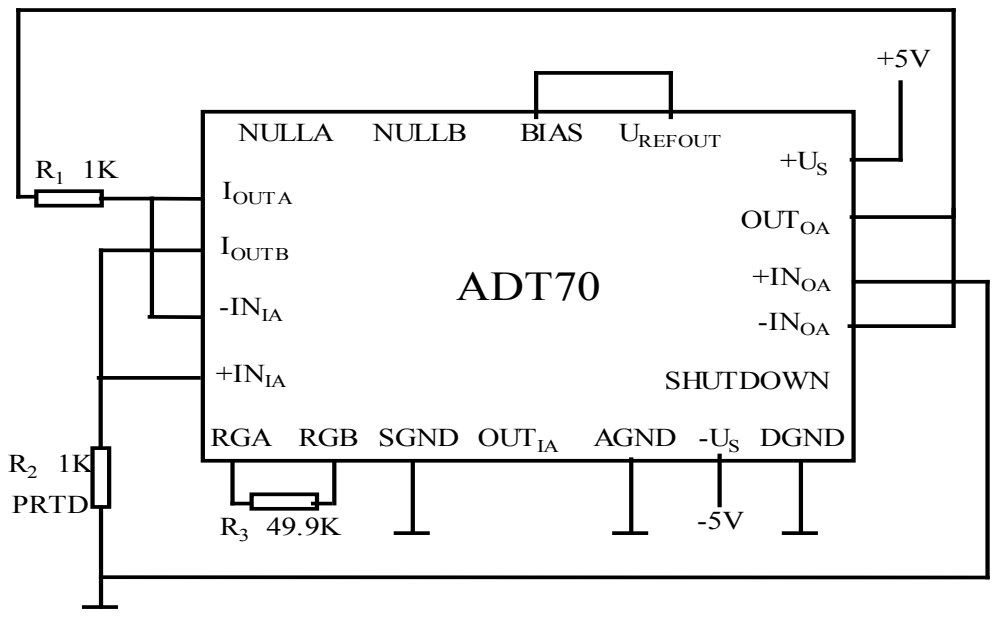

Figure 2.The sensor circuit design

The advantage of circuit design is the very close distance of platinum thermal resistance and standard resistance.There is no affects in precision differential pressure signal of long cable.

The ADT70 chip power supply provided by through the cable.

Differential voltage signal from the $\mathrm{OUT}_{\mathrm{IA}}$ be controlled by $\mathrm{MCU}$, by cable transmission to the hand-held table.

Geological survey work often in the wild far away from downtown and population.So,power consumption problem of instruments is particularly prominent.ADT70's internal chip SHUTDOWN pin can off control circuit,can be achieved micro power consumption standby state when don't work.

\subsection{Control and display circuit}

In this design,using the 5V SCM STC12C5410AD with A/Dtransfer function,to control system for the temperature sensor.STC12C5410ADis a new generation of 8051 single chip microcomputer,Its advantage ishigh speed, low power consumption, strong anti-interference.

Display module selects general instrumentation liquid crystal display module LCM045A,its working voltage is $2.7 \mathrm{~V}-5.2 \mathrm{~V}$, can display four digits, have the removable decimal point,LCD with backlight,and the backlight brightness is adjustable. 


\subsection{Power supply design}

In this design,ADT70 chip is $\pm 5 \mathrm{~V}$ power supply,STC12C5410AD chips and LCM045A also Use $+5 \mathrm{~V}$ power supply. Therefore, we need to provide $+5 \mathrm{~V}$ and $-5 \mathrm{~V}$ power supply can be realized.

In considering,we use $9 \mathrm{~V}$ alkaline batteriespower supply, use the voltage stabilizerNCP551 supply $5 \mathrm{~V}$ reference voltage, use chip ICL7660realize the output of the $-5 \mathrm{~V}$ voltage. use $\mathrm{A} / \mathrm{D}$ mental set shifting to monitor the size of the battery voltage at any time. When the voltage is low, remind instrument users replace the battery in time.

\section{Conclusion}

Platinum thermal resistance integrated data acquisition device ADT70, using an internal current source of symmetry and amplifier circuit, etc, to regulate the platinum resistance sensor signal, rule out the influence of the distance of signal transmission cable, the application of this method is suitable for remote temperature measurement.

\section{References}

1. Li Long piao, Temperature measurement system design based on the platinum resistance, Electronic technique, Practical design. 48-52.

2. Su Yuanbin, integrated signal regulator ADT70and its application, Instrumentation Technology, 2006(6): 61-62.

3. Li Shuze, Zhang Wugao, Zhang Rongrong, Huang Zhen, High precision platinum resistance temperature measurement circuit optimization design, Industrial Instrumentation and Automation, 2005(1):26-28.

4. Gong Ruikun, Li Jingyuan, Zhang Bing, The realization of high precision platinum resistance temperature measurement system, Instrumentation Technology, 2008(7):9-10.

5. B. H. Wu, N. F. Zheng, G. Fu. Small molecules control the formation of Pt nanocrystals: A key role of carbon monoxide in the synthesis of Pt nanocubes. Chemical Communications. 2011. 